\title{
Measurement of Optical Scattering Coefficient of the Individual Layers of the Human Urinary Bladder Using Optical Coherence Tomography
}

\author{
O’tega Ejofodomi \\ Department of Electrical and Electronics Engineering, Federal University of Petroleum Resources, PMB 1221, Effurun, Nigeria \\ Correspondence should be addressed to O’tega Ejofodomi; tegae@yahoo.com
}

Received 23 September 2013; Accepted 6 January 2014; Published 16 February 2014

Academic Editors: T. Nyholm and F. Rannou

Copyright (c) 2014 O'tega Ejofodomi. This is an open access article distributed under the Creative Commons Attribution License, which permits unrestricted use, distribution, and reproduction in any medium, provided the original work is properly cited.

\begin{abstract}
The author reports measurement of the optical attenuation of the urinary bladder using Optical Coherence Tomography. This method uses the exponential relationship that exists between the intensity of the back-scattered infrared light and the penetration depth. The method is applied to Optical Coherence Tomography images of the human urinary bladder and the scattering coefficients of the top three layers (urothelium, lamina propria, and muscle layers, resp.) are extracted. An optical attenuation ratio of $1: 6.2: 4.2$ for the three layers is reported.
\end{abstract}

\section{Introduction}

Bladder cancer is the fourth most common cancer among men. The current method for bladder cancer detection after the patient has reported symptoms that may be related to cancer is visual inspection of the bladder surface using endoscopic methods followed by biopsy. However, it is possible for cancerous and precancerous lesions to go undetected by urologists using conventional visual inspection. A sensitivity of $78 \%$ for bladder lesion detection using white light cystoscopy has been reported by Schmidbauer et al. [1]. Biopsies of these undetected cancer lesions are not taken simply because they are not visible to the urologist. Failure to biopsy these lesions may result in a false negative diagnosis, delaying cancer detection, and increasing mortality risk. High resolution images of the subsurface structures of the bladder will greatly enhance the urologist's ability to diagnose pathological conditions previously undetected by conventional visual inspection. Optical Coherence Tomography (OCT) has shown significant potential in this regard.

Recently, Optical Coherence Tomography (OCT), an imaging modality that measures the intensity of backscattered infrared light and produces subsurface images with micrometer resolution, has shown significant promise in early detection of bladder cancer [2-4]. The major disadvantage of OCT is the high level of scattering of nearinfrared light in biological tissues, which limits penetration depth to $1-2 \mathrm{~mm}$ [4]. However, bladder cancer tends to arise within $600 \mu \mathrm{m}$ of the tissue surface. This makes endoscopic OCT imaging systems suitable for bladder cancer detection. Several studies using OCT for detection, diagnosis, and staging of bladder cancer have been conducted and shown promising results $[3,4]$. It is still unclear how closely the changes in the optical properties of the tissue can be correlated with progression of the disease. de Reijke et al. examined human bladder tissue specimens ex vivo by $850 \mathrm{~nm}$ OCT using dynamic focusing and presented scattering coefficient data for benign bladder tissue as well as grades 1, 2, and 3 urothelial carcinoma [5].

In this paper, the author presents optical attenuation coefficients of not just the urinary bladder but of the individual layers of the normal urinary bladder.

\section{Materials and Methods}

OCT images of the normal human urinary bladder were obtained during a study conducted at the George Washington University Medical Center [6]. Images were obtained from 24 patients at high risk of having transitional-cell carcinoma 


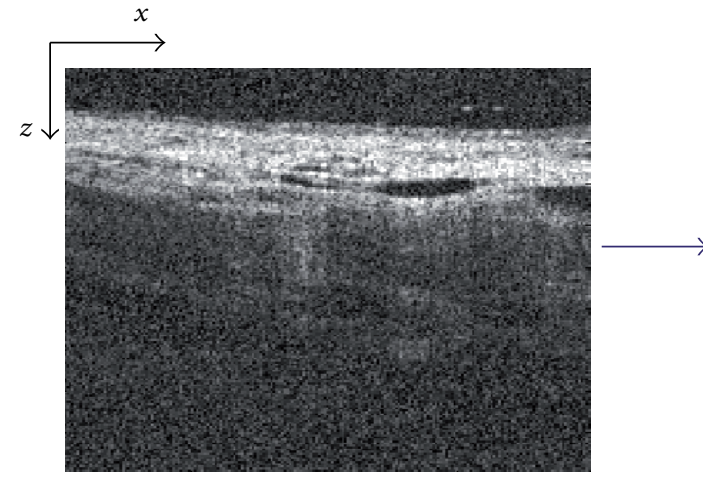

(a)

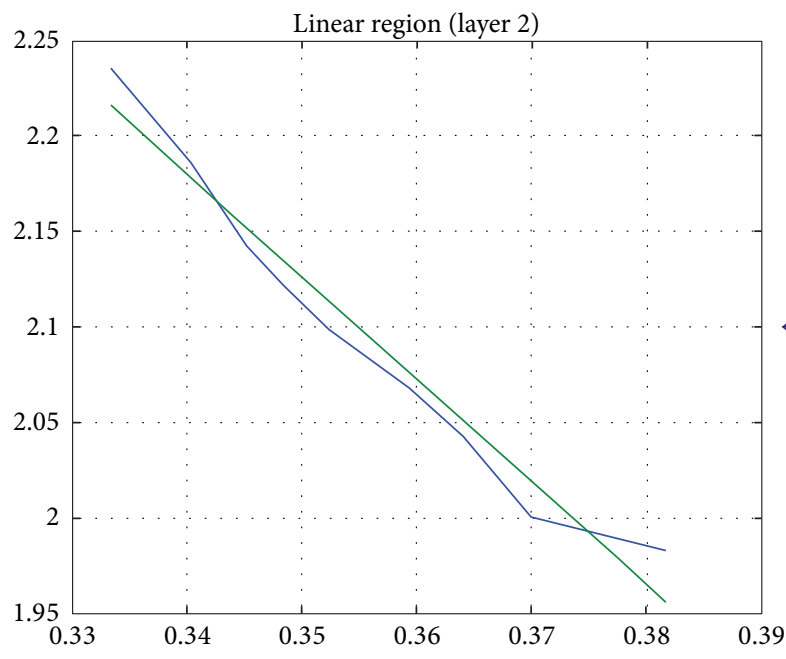

(c)



(b)

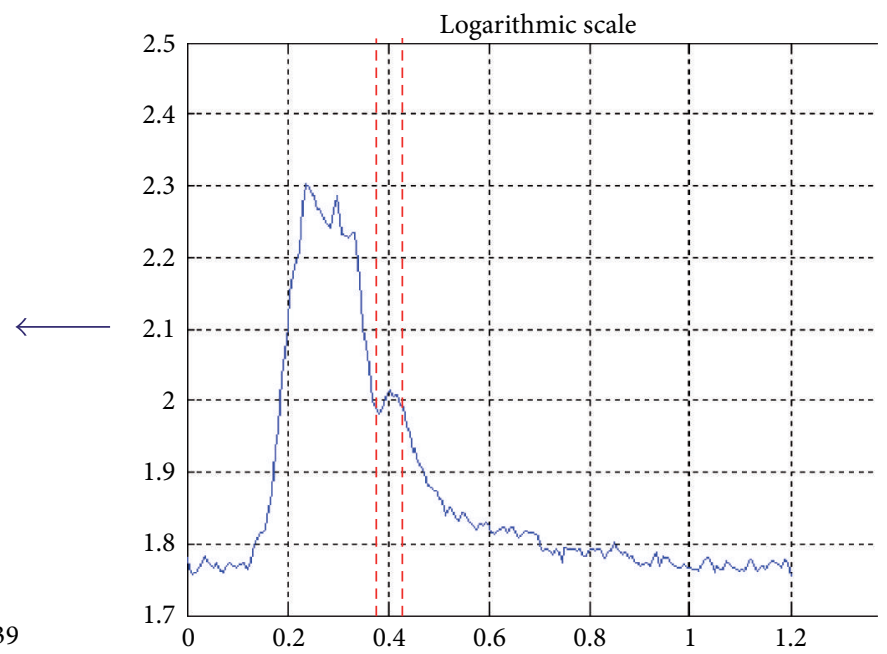

(d)

FIgURE 1: Scattering coefficient extraction algorithm. (a) Original OCT image obtained from OCT system. In (b), the A-lines are averaged and plotted as a function of depth, with intensity normalized to 1 . Natural logarithm of the intensity for the urothelium is taken in (c). User is also prompted to select linear region in (c). Linear regression analysis is performed in (d) and algorithm returns scattering coefficient, $\mu_{s}$, range of fit, $r$, and correlation coefficient, $R^{2}$.

(TCC) of the urinary bladder. These patients underwent cystoscopic examination with the OCT protocol. Scanning was performed with a $980 \mathrm{~nm} 10 \mathrm{~mW}$ superluminescent diode using a $2.7 \mathrm{~mm}(\mathrm{OD})$ optical fiber positioned through a cystoscope sheath [6]. For each urinary bladder image, the mean grayscale intensity for the urothelium, lamina propria, and muscularis layer at each depth was measured and plotted as a function of depth. The scattering coefficient is calculated by

$$
\begin{gathered}
I_{(z)}=I_{O} e^{-2 \mu_{t} z}, \\
\mu_{t}=\mu_{a}+\mu_{s},
\end{gathered}
$$

where $I_{(z)}$ is the intensity as a function of depth, $I_{O}$ is the incident intensity, $\mu_{t}$ is the total attenuation coefficient, $z$ is the penetration depth, $\mu_{a}$, is the absorption coefficient, and $\mu_{s}$ is the scattering coefficient. For most biological tissues in the near infrared region, the absorption coefficient, $\mu_{a}$ is significantly less than the scattering coefficient, $\mu_{s}$. Therefore, $\mu_{t}=\mu_{a}+\mu_{s} \approx \mu_{s}$. Graphically, $\mu_{s}$ can be obtained as the gradient of the linear region of the graph of $\ln \left(I / I_{O}\right)$ versus $z$ [7].

Figure 1 illustrates the procedure followed to extract the scattering coefficient from the OCT images, adapted from Ugryumova et al. [8]. This procedure was implemented using an algorithm implemented in MATLAB (The Mathworks, Natick, MA, USA). The algorithm has already been successfully used to adequately characterize the optical properties of bladder-wall phantoms $[9,10]$.

To reduce the effect of speckles in our calculations, we averaged over approximately $200 \mathrm{~A}$-lines [11] and the mean grayscale intensity versus depth in millimeters was plotted, as shown in Figure 1(b). The intensity values were normalized by the maximum intensity value (from the reflection at the surface of the bladder). After taking the natural logarithm of the normalized intensity, the user was prompted to select the linear region on the graph for the urothelium. Linear regression was performed on the selected region by fitting 
TABLE 1: Scattering coefficients of the human urinary bladder layers.

\begin{tabular}{lccc}
\hline Normal bladder image & Urothelium $\left(\mathrm{mm}^{-1}\right)$ & Lamina propria $\left(\mathrm{mm}^{-1}\right)$ & ${\text { Muscle layer }\left(\mathrm{mm}^{-1}\right)}^{-1}$ \\
\hline 1 & - & 0.96 & 2.25 \\
2 & - & 2.39 & 2.09 \\
3 & 0.35 & 2.69 & 0.83 \\
4 & - & 1.68 & 0.55 \\
5 & 0.31 & 2.48 & 1.19 \\
\hline Average $\mu_{s}$ & 0.33 & 2.04 & 1.38 \\
$\mu_{s}$ standard deviation & 0.03 & 0.71 & 0.75 \\
Average correlation coefficient $R^{2}$ & 0.62 & 0.94 & 0.97 \\
\hline
\end{tabular}

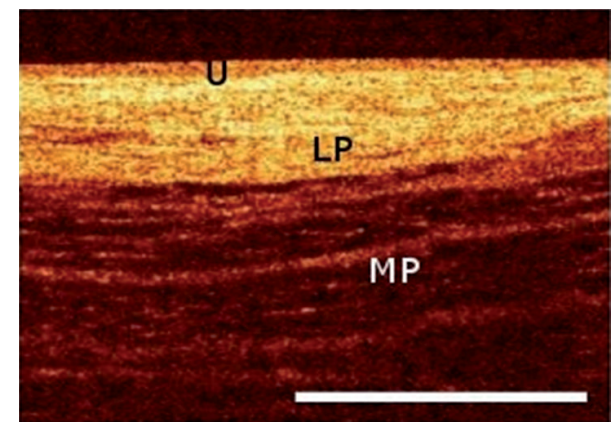

(a)

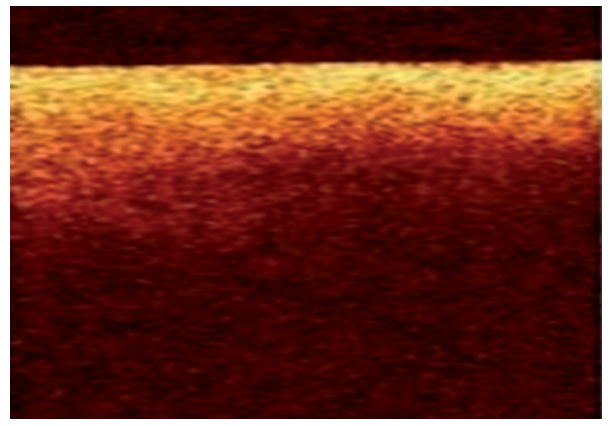

(b)

FIGURE 2: OCT images of the bladder. (a) Normal bladder showing a thin urothelium (U) over a bright lamina propria (LP). The muscularis propria (MP) is below the lamina propria. (b) Invasive cancer of the bladder. The structures of the urothelium, lamina propria, and muscularis are indistinguishable.

the data of the region to a polynomial that best fits the data. After performing a linear regression analysis on the selected region, the equation of the linear fit, the scattering coefficient, $\mu_{s}$, and the correlation coefficient, $R^{2}$, were extracted. The results from the data calculation as well as the graphs were then exported and stored as text files and bitmap images, respectively. This process was repeated for the lamina propria and the muscularis layer.

\section{Results}

The first three layers of the urinary bladder are the urothelium, lamina propria, and the muscle layer. The scattering coefficients for each of these layers are shown in Table 1. We were unable to obtain urothelium $\mu_{s}$ for 3 of the 5 images. This layer was less than $100 \mu \mathrm{m}$ and so it did not permit consistent accurate linear regression analysis. The scattering coefficients of the urothelium, lamina propria, and muscle layers were $0.33 \pm 0.03 \mathrm{~mm}^{-1}, 2.04 \pm 0.71 \mathrm{~mm}^{-1}$, and $1.38 \pm 0.75 \mathrm{~mm}^{-1}$, respectively, giving rise to a ratio of $1: 6.2$ : 4.2.

Figures 2(a) and 2(b) show OCT images of a normal human urinary bladder, and a cancerous urinary bladder respectively. Studies that have been performed have shown that the optical properties of the urinary bladder are altered as a result of the presence of cancer $[4,6]$. In Figure 2(a), the uroethlium, lamina propria, and muscularis layers can be distinguished optically. However, in Figure 2(b) with invasive cancer, the three layers are indistinguishable optically.

The normal bladder in Figure 2(a) shows a thin urothelium (U) over a bright lamina propria (LP). There is a well-demarcated border, with good contrast, between the urothelium and the lamina propria. The muscularis propria (MP) is below the lamina propria, and once again the border is well demarcated and there is good contrast between the layers. In Figure 2(b), the layers of the cancerous urinary bladder are indistinguishable. This likely represents cancer that has invaded into and possibly through the muscularis [12].

The results presented in this paper characterize only the optical properties of the top three layers of the normal human urinary bladder. However, since the optical properties of the urinary bladder change with the onset and progression of cancer, the results presented in this paper would be useful in forming a standard against which changes in the optical properties of each layer due to cancer can be measured.

\section{Conclusion}

The optical scattering coefficients of the urothelium, lamina propria, and muscularis layers of the normal human urinary bladder have been measured. The scattering coefficients of the urothelium, lamina propria and muscle layers were $0.33 \pm$ 
$0.03 \mathrm{~mm}^{-1}, 2.04 \pm 0.71 \mathrm{~mm}^{-1}$, and $1.38 \pm 0.75 \mathrm{~mm}^{-1}$, respectively, giving rise to a ratio of $1: 4.2: 2.8$. Since the presence of cancer alters the optical properties of these layers, future work will include measurements of the optical properties of cancerous bladder images to determine the relationship between the changes in the optical scattering coefficient and the onset and progression of cancer in the human urinary bladder.

\section{Conflict of Interests}

The authors declare that there is no conflict of interests regarding the publication of this paper.

\section{References}

[1] J. Schmidbauer, F. Witjes, N. Schmeller, R. Donat, M. Susani, and M. Marberger, "Improved detection of urothelial carcinoma in situ with hexaminolevulinate fluorescence cystoscopy," Journal of Urology, vol. 171, no. 1, pp. 135-138, 2004.

[2] Y. T. Pan, Z. G. Wang, Z. L. Wu, A. Jain, and H. K. Xie, "Diagnosis of biological tissue morphology and function with endoscopic optical coherence tomography," in Proceedings of the IEEE 27th Annual International Conference of the Engineering in Medicine and Biology Society (EMBS '05), pp. 7217-7220, September 2005.

[3] M. J. Manyak, N. D. Gladkova, J. H. Makari et al., "Evaluation of superficial bladder transitional-cell carcinoma by optical coherence tomography," Journal of Endourology, vol. 19, no. 5, pp. 570-574, 2005.

[4] C. A. Lingley-Papadopoulos, M. H. Loew, M. J. Manyak, and J. M. Zara, "Computer recognition of cancer in the urinary bladder using optical coherence tomography and texture analysis," Journal of Biomedical Optics, vol. 13, no. 2, Article ID 024003, 2008.

[5] T. M. de Reijke, M. Visser, J. de la Rosette, T. G. van Leeuwen, and E. C. Cauberg, "Quantitative measurement of attenuation coefficients of bladder biopsies using optical coherence tomography for grading urothelial carcinoma of the bladder," Journal of Biomedical Optics, vol. 15, no. 6, Article ID 066013, 2010.

[6] M. J. Manyak, N. D. Gladkova, J. H. Makari et al., "Evaluation of superficial bladder transitional-cell carcinoma by optical coherence tomography," Journal of Endourology, vol. 19, no. 5, pp. 570-574, 2005.

[7] J. M. Schmitt, A. Knuttel, and R. F. Bonner, "Measurement of optical properties of biological tissues by low-coherence reflectometry," Applied Optics, vol. 32, no. 30, pp. 6032-6042, 1993.

[8] N. Ugryumova, S. J. Matcher, and D. P. Attenburrow, "Measurement of bone mineral density via light scattering," Physics in Medicine and Biology, vol. 49, no. 3, pp. 469-483, 2004.

[9] O. A. Ejofodomi, V. Zderic, and J. M. Zara, "Tissue-mimicking bladder wall phantoms for evaluating acoustic radiation forceoptical coherence elastography systems," Journal of Medical Physics, vol. 37, no. 4, pp. 1440-1448, 2010.

[10] O. A. Ejofodomi, V. Zderic, and J. M. Zara, "Optical/acoustic radiation imaging in tissue-mimicking bladder wall phantoms," in Proceedings of the 8th IEEE International Symposium on Biomedical Imaging: From Nano to Macro (ISBI '11), pp. 524-527, April 2011.
[11] J. M. Schmitt, S. H. Xiang, and K. M. Yung, "Speckle in optical coherence tomography," Journal of Biomedical Optics, vol. 4, no. 1, pp. 95-105, 1999.

[12] Imalux Corporation, January 2013, http://www.imalux.com/. 

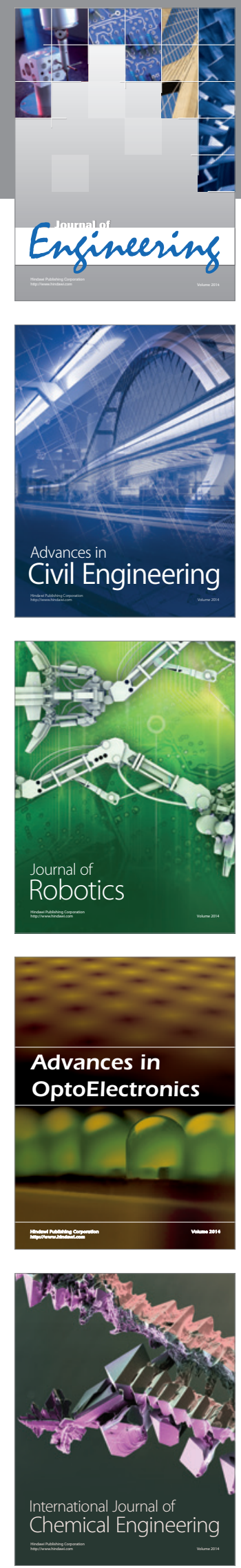



The Scientific World Journal


Submit your manuscripts at http://www.hindawi.com
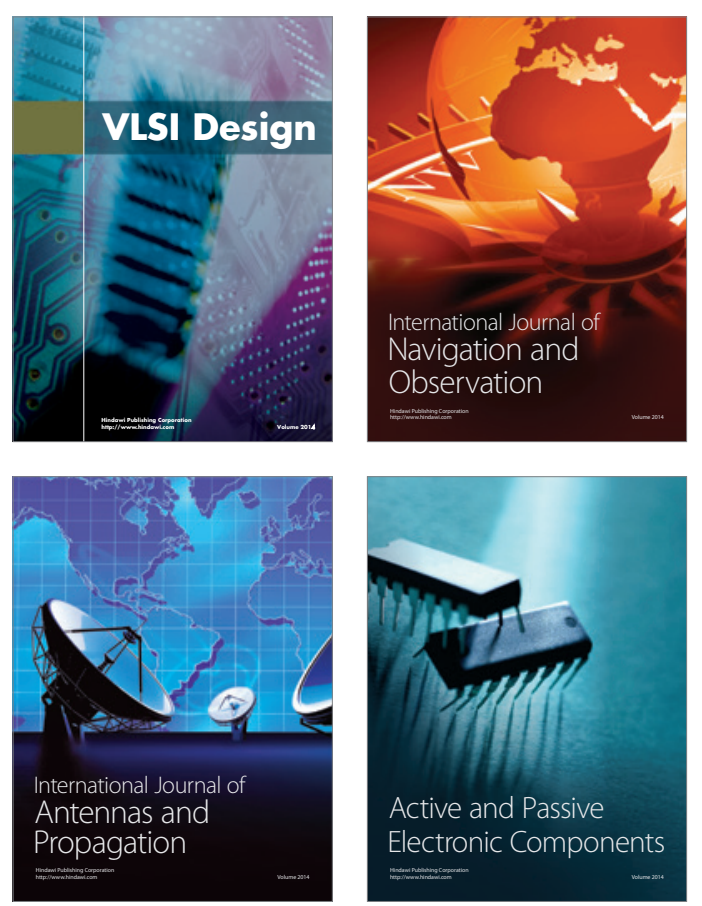
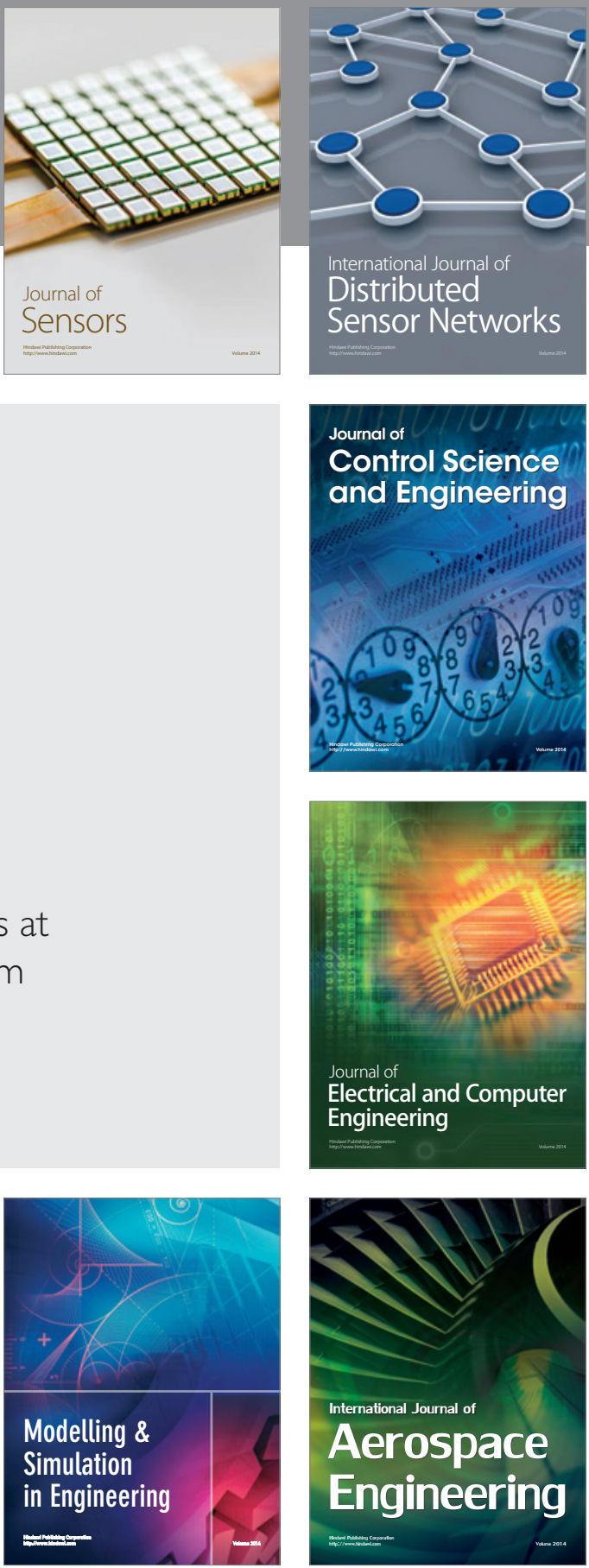

Journal of

Control Science

and Engineering
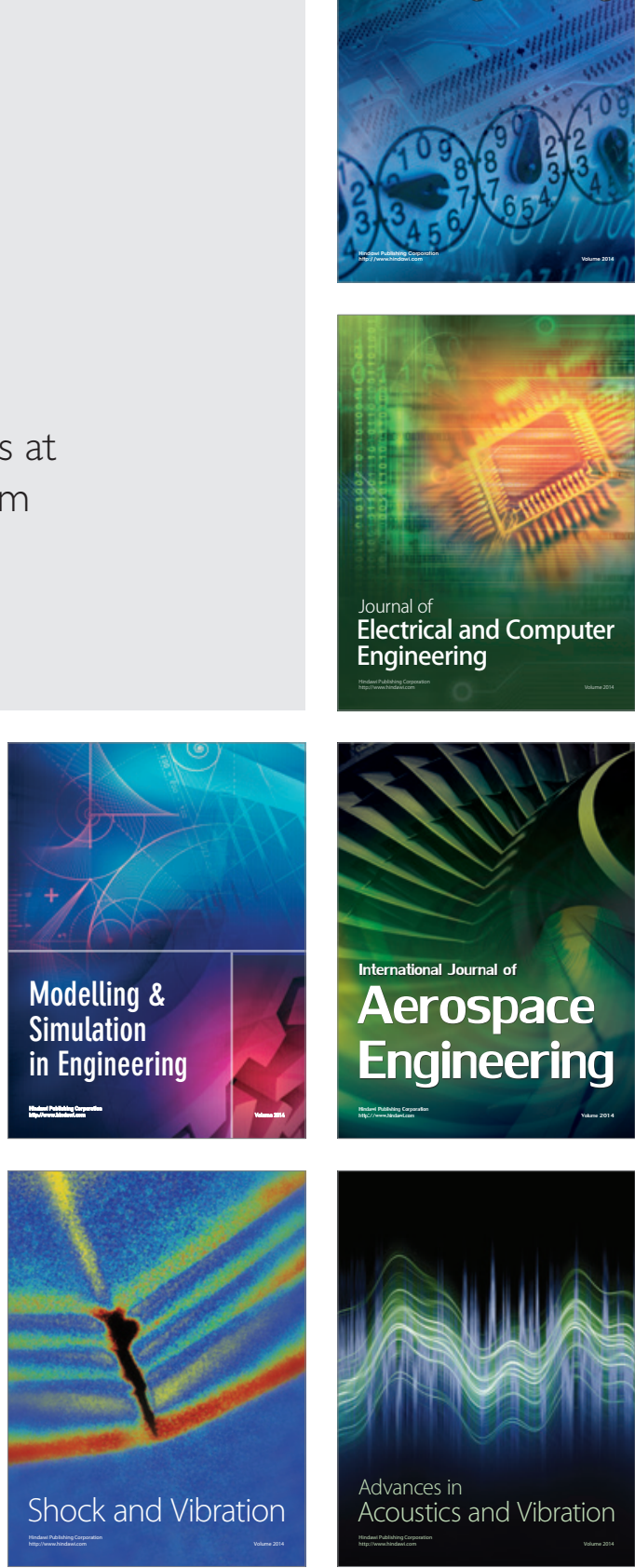